\section{Historia de la}

enseñanza en

Colombia:

\section{entre saberes}

\section{y disciplinas escolares}

\section{História do ensino na Colômbia: entre saberes e disciplinas escolares}

\section{A History of Teaching in Colombia: Between School Knowledges and Disciplines}

\section{Resumen}

El artículo desarrolla algunas ideas y resultados del proyecto de investigación "Saberes y disciplinas escolares en Colombia", que se trazó como objetivo: analizar históricamente desde la relación pedagogía-ciencias-saberes, el proceso de configuración del campo de las disciplinas y saberes escolares en Colombia, con el objeto de hacer visibles las formas de apropiación de las ciencias y los saberes en los diversos niveles de la enseñanza en Colombia. En sus apartados despliega las orientaciones conceptuales y teóricas del proyecto de investigación, a través de las reflexiones que surgieron en torno a las nociones de saber escolar y disciplina escolar. Continúa con el desarrollo y la descripción de las herramientas metodológicas utilizadas en la investigación, para cerrar con algunas reflexiones que buscan mostrar los desafíos que implica hacer historia de la enseñanza desde la perspectiva de los saberes y las disciplinas escolares.

\section{Palabras clave}

Saber escolar, disciplina escolar, saber pedagógico, enseñanza, escuela, enseñanza en Colombia

\section{Resumo}

0 artigo desenvolve algumas ideias e resultados do projeto "Saberes e disciplinas escolares na Colômbia", que traçou como objetivo: analisar historicamente desde a relação pedagogia-ciência-saberes, o processo de configuração do campo das disciplinas e saberes escolares na Colômbia, com o objetivo de fazer visíveis as formas de apropriação das ciências e os saberes nos diversos níveis do ensino na Colômbia. Em seus assuntos espalha as orientações conceptuais e teóricas do projeto de pesquisa, a traves das reflexões que surgiram em volta às noções de saber escolar e disciplina escolar. Continua com o desenvolvimento e a descrição das ferramentas metodológicas utilizadas na pesquisa, para fechar com algumas reflexões que procuram mostrar os desafios que implica fazer história do ensino, desde a perspectiva dos saberes e as disciplinas escolares.

\section{Palavras chave}

Saber escolar, disciplina escolar, saber pedagógico, ensino, escola, ensino na Colômbia

\section{Abstract}

This article develops some ideas and results from the research project "School Knowledges and Disciplines in Colombia." The project attempted to analyze historically, from the relationship between pedagogy, science and knowledges, how school knowledges and disciplines were shaped in Colombia, in order to highlight the ways of appropriation of science and knowledges at different teaching levels. The study shows the conceptual and theoretical orientations of the research, reflecting upon the notions of school knowledge and school disciplines. After developing and describing the methodological tools used during the research, it presents some reflections on the challenges of studying the history of teaching from a school knowledge and discipline approach.

\section{Key words}

School knowledge, school discipline, pedagogical knowledge, teaching, school, teaching in Colombia

\section{Rafael Ríos Beltrán*}

* Doctor en Educación por la Universidad de Antioquia. Profesor titular en el Instituto de Educación y Pedagogía de la Universidad del Valle. Correo electrónico: rafael.rios@correounivalle.edu.co

Fecha de recepción: Abril 8 de 2015 Fecha de aprobación: Mayo 22 de 2015 


\section{Introducción}

S e puede afirmar que el estudio sobre los contenidos de enseñanza de las disciplinas científicas y saberes en Colombia es una novedad, un camino por abrir. Ya lo había dicho Durkheim en sus clases de Pedagogía en la Universidad de la Sorbonne en París: "una historia de la enseñanza es la mejor de las pedagogías" (1992, p. 34).

El campo de la historia de los saberes y disciplinas escolares tiene como objeto de estudio los contenidos de enseñanza. Este campo se ocupa de analizar y reflexionar sobre cómo circulan y se apropian en la escuela la enseñanza de las ciencias y los saberes. En el ámbito internacional, se conocen tres versiones diversas en Europa representadas por Goodson Ivor en Inglaterra, André Chervel y Dominique Julia en Francia, y Antonio Viñao y Raimundo Cuesta en España. De acuerdo con Chervel, la historia de los contenidos disciplinares es su componente central, el eje alrededor del cual se va constituyendo este campo de estudios sobre la enseñanza.

Quizás nos adentremos aún más en terrenos poco habitados por la reflexión y el análisis de la enseñanza en Colombia si la miramos desde la perspectiva de la historia de los saberes y disciplinas escolares. Pensamos que abrir la reflexión desde esta perspectiva histórica sea uno de los caminos para hacer visible la relación pedagogía-ciencia-saberes a través de la enseñanza. De acuerdo con esto, el artículo se divide en tres partes: la primera desarrolla las orientaciones conceptuales y teóricas del proyecto de investigación, a través de las cavilaciones que surgieron en torno a las nociones de saber escolar y disciplina escolar. En la segunda parte se desarrollan algunas reflexiones en torno a las herramientas metodológicas utilizadas en la investigación. La tercera parte presenta algunas reflexiones finales y nuevas perspectivas de análisis con respecto a la historia de la enseñanza de las ciencias y los saberes.

\section{Problematización: orientaciones conceptuales y teóricas}

En palabras del francés André Chérvel, las disciplinas escolares constituyen un conjunto cultural muy original ya que

... los contenidos de la enseñanza se conciben como entidades sui generis, independientes hasta cierto punto de cualquier realidad cultural ajena a la escuela y dotadas de una organización, una economía propia y una eficacia que sólo parecen deber a sí mismas, es decir, a su propia historia. (1991, p. 63)
A lo que añade:

... según la opinión común, la escuela enseña aquellas ciencias que han demostrado ser eficaces en otros campos. Enseña la gramática porque ésta, que es una creación secular de los lingüistas, dice la verdad sobre la lengua, enseña ciencias exactas, como las matemáticas y. cuando se orienta hacia las matemáticas modernas, se piensa es que porque se ha producido una revolución en el mundo de las ciencias matemáticas; enseña, en fin, la historia de los historiadores, la civilización y la cultura latinas de la Roma antigua, la filosofía de los grandes filósofos, el inglés que se habla en Inglaterra o en Estados Unidos y el francés de todo el mundo. La imagen que se tiene en general de la pedagogía tiene una relación directa con este concepto de las enseñanzas escolares. Si las disciplinas escolares se relacionan directamente con las ciencias, con los saberes, con los conocimientos técnicos vigentes en la sociedad global, las diferencias entre unas y otras se atribuyen entonces a la necesidad de simplificar, e incluso de vulgarizar, para un público joven conocimientos que no pueden proponérsele en estado puro ni en su integridad. Se estima, por tanto, que la labor de los pedagogos consiste en desarrollar aquellos "métodos" que permitan a los alumnos asimilar cuanto antes y en las mejores condiciones la máxima porción posible de la ciencia de que se trate. En esta hipótesis, pues, las disciplinas se reducen a simples "metodologías": por lo demás, es éste el término con que se designa la pedagogía en Bélgica, e incluso y a veces en Francia. Paralelamente a la disciplina —vulgarización, se ha impuesto la imagen de la pedagogía- lubricante, encargada de engrasar los mecanismos y conseguir que la máquina funcione. (1991, p. 64)

El interés por abrir una perspectiva de análisis histórico de los saberes y las disciplinas escolares en Colombia no radica en mostrar el camino hacia la pedagogía, entendida como aquel proceso que nos señala cómo enseñar y a través de qué lubricantes y mecanismos ha funcionado la máquina de la enseñanza de las ciencias y los saberes en la escuela colombiana, ni tampoco para señalar estas ciencias como fines en sí mismos, y como conocimientos necesarios para ser transmitidos en la escuela, mediante métodos eficaces.

Por el contrario, de una parte, y siguiendo a Olga Lucia Zuluaga (1999), nos interesa problematizar esa imagen de la pedagogía reducida a la manera de enseñar y al concepto de método. Bien sabemos que sin una mirada juiciosa de los otros objetos de la pedagogía no es posible aproximarnos a los diversos modos de configuración del sujeto, la enseñanza, la escuela, el maestro y la cultura escolar. 
De otra parte, y siguiendo las orientaciones de John Dewey, nos interesa problematizar también el carácter dualista como se ha venido entendiendo las relaciones entre pedagogía-ciencia-saberes. Con Dewey no nos limitaremos al debate sobre las ciencias y su enseñanza en la escuela. En efecto, una lectura de la enseñanza de las ciencias y los saberes en el saber pedagógico colombiano desde la línea de investigación histórica de las disciplinas escolares, implica romper con las clásicas problemáticas y tensiones entre las ciencias de referencia y su enseñanza.

El problema residiría en sus formas de apropiación. Para Dewey deben desarrollarse nuevas formas de apropiación de lo científico en la escuela:

... nuevas formas de integrar el material científico y de darle usos no previstos y no familiares. Cuando, en pedagogía, el psicólogo u observador o experimentador en cualquier campo reduce sus hallazgos a una regla a ser adoptada de manera uniforme, entonces, y sólo entonces, se presenta un resultado objetable y destructivo para el libre juego de la pedagogía como arte. $(1929$, p. 6$)$

Esto nos conduce a pensar los saberes y las disciplinas escolares como formas de apropiación de la ciencia y los saberes en la escuela. Aquí las prácticas de enseñanza no solo harían visible procesos de institucionalización y de funcionamiento de la ciencia y los saberes en las instituciones educativas, sino también comprenderían las formas de enunciación, circulación y apropiación de los saberes enseñados.

Lo anterior permitió trabajar con la siguiente hipótesis: los saberes y disciplinas escolares poseen una autonomía constitutiva y son, en consecuencia, una invención de las prácticas de enseñanza. Esta condición los distancia de la tradición escolar moderna que configura a la escuela como un espacio subordinado de las ciencias y las políticas educativas, a la enseñanza como una técnica derivada de estas, y al maestro como un sujeto que aplica mediante métodos efectivos y eficientes dichas ciencias y políticas.

Pero a lo que se apostó en el proyecto no fue a mirar disciplinas y saberes, sino saberes y disciplinas escolares. Entonces, ¿qué es lo que en la escuela se transforma en un saber y una disciplina escolar? Según Chervel, hay dos disciplinas: la escolar (Ciencias Naturales, Ciencias Sociales, Educación Física) y otra es una disciplina que también se pone a funcionar en la escuela, aquella que remite al comportamiento, a eso también se le llama disciplina. Citemos al mismo autor:

En el ámbito escolar, el término disciplina y la expresión disciplina escolar sólo designaban, hasta finales del siglo XIX, la policía de los centros docentes, la represión de las conductas susceptibles de alterar el orden establecido y aquella parte de la educación de los alumnos que debía contribuir a ello. En el sentido que aquí nos interesa de contenidos de la enseñanza, el término brilla por su ausencia en todos los diccionarios del siglo xIX, y no aparece ni siquiera en el Diccionario de la Academia de 1932. ¿Cómo se designaban entonces antes de dicha época, los diferentes órdenes de enseñanza? ¿Cuál era el título general que se daba a las rúbricas de los distintos cursos? (Chervel, 1991, p. 59).

En el contexto del proyecto se entiende la disciplina como la ha trabajado la profesora Zuluaga, siguiendo a Foucault, en el orden del saber, como forma de producción y control de nuevos discursos. De ahí que se analice la pedagogía como la disciplina que conceptualiza, aplica y experimenta los conocimientos referentes a la enseñanza de los saberes específicos, en las diferentes culturas. Sin embargo, sabemos que la pedagogía no es la enseñanza sino una práctica cuyo campo de aplicación es el discurso (1999).

Al definir la pedagogía como disciplina, en el orden del saber, Zuluaga pretende hacer visible su potencia para enfrentar su reduccionismo a lo instrumental y metódico y para responder a las demandas de las ciencias respecto de los métodos de enseñanza. En consecuencia, la pedagogía se toma como

... el discurso que posibilita al maestro ser el soporte de un saber específico circunscrito a las prácticas que tienen lugar en las prácticas de saber, y como el lugar teórico que conceptualiza tanto acerca de la enseñanza como de las múltiples relaciones conceptuales y prácticas con las cuales entra en relación la enseñanza. Relaciones escuela-sociedad; enseñanza-ciencia; enseñanza-cultura, formación del hombre-conocimiento y cultura; formación del hombre-conocimiento-sociedad, etc. (1999, p.14)

Zuluaga no utiliza la expresión saberes escolares, ni menos disciplinas escolares, al modo francés e inglés, retomando las tradiciones abiertas por Chervel y Julia e Ivor Goodson, las cuales se ocupan de analizar históricamente los contenidos de enseñanza en la escuela. Por el contrario, plantea el análisis de la enseñanza a través de la noción práctica pedagógica. Para la autora:

Rescatar la práctica pedagógica significa en su sentido más amplio: recuperar la historicidad de la Pedagogía, tanto para analizarla como saber, como para analizar sus procesos de formación como disciplina, trabajar con la discursividad de la Pedagogía y analizar la práctica del saber pedagógico en nuestra sociedad. Entiendo por historicidad de la Pedagogía, el carácter positivo tanto de las fuentes como de la discursividad acerca de la enseñanza. Discursividad 
que registra no sólo objetos de saber sino también nociones, conceptos y modelos que dan cuenta de la búsqueda de sistematicidad de la Pedagogía. Distingo entre el análisis de la Pedagogía como saber y el análisis de sus procesos de formación como disciplina, para indicar que el saber no está referido solamente al pasado sino también al presente; en este sentido cubre la cotidianidad de la enseñanza, del maestro y de la escuela, en la actualidad. (1999, p. 12)

Para los investigadores de este proyecto la noción de práctica pedagógica sigue siendo estratégica para nombrar los saberes y las disciplinas que circulan, se apropian y se producen en la escuela. Por eso la importancia de reconocer en la enseñanza su estatuto de práctica de saber entre prácticas. Para Zuluaga, La historia de la práctica pedagógica posibilita el análisis de las "formas de lo dicho", ya que en las prácticas de saber en la enseñanza, la Pedagogía produce "formas" de enunciación de los saberes. "Sólo un estudio de carácter histórico puede mostrar el lugar que ha ocupado la práctica pedagógica entre las prácticas de saber" (1999, pp. 15-16).

En efecto, el proyecto se ha trazado como objetivo analizar históricamente desde la relación pedagogíaciencias-saberes, el proceso de configuración del campo de las disciplinas y los saberes escolares en Colombia, con el fin de hacer visibles las formas de apropiación de las ciencias y los saberes en los diversos niveles de la enseñanza en Colombia.

\section{¿Saberes y/o disciplinas escolares?: comentarios y reflexiones}

Bien sabemos que en la escuela podemos encontrarnos con discursos en estados de positividad ${ }^{1}$ o no, es decir, un conjunto de elementos, objetos, formas de enunciación, conceptos y estrategias, formados de manera regular por una práctica discursiva, conjunto que Michel Foucault llamaba saber, sin excluir la ciencia o la no-ciencia.

En ese sentido, por una parte, consideramos que ha sido un acierto reflexionar conjuntamente sobre saberes y disciplinas escolares, y no bajo la expresión disyuntiva de saberes o disciplinas, porque nos evita ambigüedades respecto de si hacemos una historia de la enseñanza de los saberes y disciplinas escolares en Colombia, o una historia epistemológica de la ense- ñanza de las disciplinas y las ciencias en la escuela ${ }^{2}$. De ahí la importancia en términos metodológicos y teóricos de que agregáramos la palabra "escolares" a las nociones de saber y disciplina.

De otra parte, entendemos que para analizar y enunciar saberes y disciplinas escolares debemos trabajar más en función de las áreas de enseñanza, porque en la escuela funcionan y se enseñan saberes y disciplinas que no tienen nada que ver con la disciplina de referencia, ya que dependen del campo especifico de las prácticas y de los sujetos de la enseñanza. En esta dirección, Zuluaga ya había señalado en Pedagogía e Historia lo siguiente:

\begin{abstract}
Se tiene la costumbre de aplicar en un campo de saber para resolver problemas concretos, modelos teóricos de otros campos del saber, sin recurrir a la trama conceptual e histórica del saber al que corresponde tal problema concreto; pero cuando se trata de resolver un problema tan complejo como el de la enseñanza de las diferentes ciencias, problema que involucra a la Pedagogía y que incumbe tanto a los maestros como a los profesores, no queda otra salida que volver a la Pedagogía, asumiéndola en su historicidad, de lo contrario las preguntas y soluciones carecerían de territorialidad. (1999, p. 48. Énfasis fuera del original)
\end{abstract}

Para los maestros, se trata del saber que les confiere identidad intelectual para relacionarse con los saberes enseñados, con la ciencia y con los intelectuales de otros saberes. Para los profesores se trata de la Pedagogía como una disciplina auxiliar en la enseñanza de una ciencia o disciplina (Zuluaga, 1999).

Por ejemplo, si se examinan los manuales escolares de Física, Ciencias Naturales, Matemáticas y Filosofía estamos en el territorio de las disciplinas y saberes escolares, porque el manual está conectado y configurado en el tejido de las prácticas de enseñanza. El manual escolar tiene allí por elementos constitutivos:

\footnotetext{
El saber pedagógico, la práctica política, la política de los saberes, el maestro, la institución escolar, los alumnos y otros funcionarios de la práctica pedagógica. [...] ellos aparecen como elementos articulados a la práctica en su globalidad, pero también se manifiestan como articuladores de configuraciones sectoriales y específicos de la práctica. (Zuluaga, Martínez, 1997, p. 2, 3)
}

1 Foucault utiliza el término "positividad" para referirse al análisis discursivo de los saberes desde un punto de vista arqueológico. Determinar la positividad de un saber no consiste en referir los discursos a la totalidad de la significación ni a la interioridad de un sujeto, sino a la dispersión y la exterioridad (Castro, 2004, p. 272).
2 En este sentido, ya Olga Lucia Zuluaga había realizado una distinción similar para el análisis de la Pedagogía -que además fue inspiradora para tal distinción y uso de la expresión saberes y disciplinas con el mote de "escolares"- en el apartado de su libro Pedagogía e historia (1999) con la pregunta "¿Historia epistemológica de la pedagogía o historia del saber pedagógico?”. 
De ahí se comprende el por qué en este estudio se analiza históricamente la enseñanza de las disciplinas y los saberes escolares más allá de un problema epistemológico, ya que no nos interesa una historia interna de la verdad sobre la enseñanza de las ciencias y las disciplinas en la escuela, sino una historia de la enseñanza desde la perspectiva del saber, que nos permita situar las diversas prácticas de enseñanza en la escuela, externas e internas, frente a la verdad de las ciencias y las disciplinas de referencia. Para Foucault, hay dos tipos de historias de la verdad:

La primera es una especie de historia interna de la verdad, que se corrige partiendo de sus propios principios de regulación: es la historia de la verdad tal como se hace en o partir de la historia de las ciencias. Por otra parte, creo que en la sociedad, o al menos en nuestras sociedades, hay otros sitios en los que se forma la verdad, allí donde se definen un cierto número de reglas de juego, a partir de las cuales vemos nacer ciertas formas de subjetividad, dominios de objeto, tipos de saber y, por consiguiente, podemos hacer a partir de ello una historia externa, exterior, de la verdad. (1995, p. 17)

De acuerdo con esto, en la enseñanza de las ciencias sociales en Colombia se encuentra más una historia cercana al segundo tipo de verdad que se acaba de describir con Foucault, ya que cuando se analizan las prácticas de enseñanza de las ciencias sociales se hace visible cómo el enunciado "ciencias sociales" que funciona en la escuela no proviene de unas disciplinas universales previamente establecidas, sino de saberes e intereses locales que la escuela selecciona, crea y considera pertinentes para su enseñanza.

A partir del trabajo de Alejandro Álvarez sobre las ciencias sociales en Colombia, sabemos que la escuela desde los inicios del siglo $\mathrm{xx}$ abandona el proyecto universal que le caracterizaba, para pasar a ser una institución más local que produce los diversos sentidos sobre la identidad nacional. En consecuencia:

El discurso pedagógico de la época estuvo ligado a la necesidad de producir una ciencia nacional, un saber y un sistema de verdades propios. Los saberes sobre el pasado, el pueblo y el territorio, componentes de la nación, se convirtieron antes que nada en disciplinas escolares, así la escuela resultó estratégica para conseguir el efecto de verdad que dichas nociones necesitaban. Los métodos de enseñanza activa debían ayudar a forjar una identidad ligada al pasado nacional para construir un sujeto territorializado y para crear allí una conciencia de pueblo. [...] Lo que se llamó territorialización del sujeto - que operó a través de la reforma educativa nacionalista y la consiguiente incorporación de la pedagogía activa - planteaba que los estudiantes debían relacionarse en forma directa con las cosas, las personas, los fenómenos naturales y sociales, aquello que configuraba la realidad, lo cual dio origen a un campo disciplinar llamado ciencias sociales. (2013, pp. 10, 11)

Ahora bien, ¿la triada escribir-leer-contar puede denominarse saberes fundacionales en el contexto escolar, puesto que estos procesos han dado un sentido de existencia a la escuela como institución centrada en la enseñanza? Para el historiador francés Jean Hebrard (1989) esta triada funciona como destrezas y habilidades sin ningún respaldo en las disciplinas académicas y su jerarquía.

Desde la emergencia de la escuela en Colombia en la época colonial, hasta nuestros días la aritmética, la lectura y escritura, por excelencia, han sido los saberes que han permanecido en el centro de cualquier reforma curricular o plan de estudios (Martínez Boom, 1986). Si bien estos saberes son exógenos a la institución escolar, es allí donde histórica y pedagógicamente han sido ampliamente difundidos y han adquirido un estatuto que los diferencia de otros saberes o disciplinas. Así lo demuestran las reflexiones sobre la enseñanza de la escritura y la lectura desarrolladas por los investigadores del proyecto. Por una parte, Sandra Herrera considera que:

La escritura es un saber constitutivo de la escuela desde su emergencia en la colonia y se constituye, desde el análisis de Foucault "como una pieza esencial en los engranajes de la disciplina”. Cuando nos referimos aquí a la escritura no hablamos de la función comunicativa que ésta tiene, nos referimos a la técnica que implica un control del cuerpo en las posturas, los utensilios, los ejercicios y su repetición constante. Es decir, nos referimos a la disciplinización del cuerpo mediante unas técnicas de rutinas y coordinación de los movimientos. Para ser exactos nos estamos refiriendo a un saber, por muchos autores definido como "menor" en las artes, que tuvo y aún conserva una existencia por fuera de la escuela, fue apropiada como un saber escolar que fue enseñado y utilizado con un propósito disciplinar, pero que con el proceso de modernización de esta institución y de los saberes escolares, terminó subsumido como una parte de la escritura. $(2015$, p. 2)

De otra parte, Lina Quintana indaga por las escenas de lectura ${ }^{3}$ para hacer visible la configuración de un saber en la escuela. $\mathrm{Al}$ respecto afirma:

3 La escena de lectura es una noción de análisis para hacer historia de la lectura y la escritura tomada del grupo Histelea de Argentina, liderado por el profesor Rubén Cucuzza, para él la escena de lectura es el lugar donde se realiza y materializa lo escrito como práctica social de comunicación. Véase Rubén H. Cucuzza (2008). Retórica de las escenas de lectura en las 
La noción de escena de enseñanza de la lectura permite en el campo pedagógico mostrar a través de los manuales, tesis, ponencias, conferencias radiales que escribieron los maestros, la manera como ellos idearon y crearon prácticas de enseñanza de la lectura en la escuela y también cómo dichas prácticas de enseñanza de lectura constituyen un saber que se produjo en la escuela, un saber que no era prescrito, sino que obedecía a lo que aconteció en ella por acción del maestro, unas formas de hacer y de formar. Se trata de visibilizar prácticas de saber que fueron acumulando conceptos y procedimientos pedagógicos en el espacio escolar en la primera mitad del siglo xx en Colombia para reconocer una tradición pedagógica en el país. No se trata aquí de abonar a la polaridad de lo que es tradicional o nuevo, se trata de describir históricamente la identidad de los maestros como una comunidad que permitió por un lado, ver la pedagogía funcionando como un saber, donde se privilegian las formas y no las normas, y por el otro se delinea, muestra y configura un sujeto lector infantil con unas características específicas. (2015, p. 2)

De las matemáticas, la escuela apropia y desarrolla lo referido a la aritmética para completar la triada "leer, escribir y contar". De acuerdo con esto, conocer la aritmética consiste en dominar los algoritmos de las cuatro operaciones y saber aplicarlos para resolver problemas cotidianos de la vida social. Así lo demostró el manual de enseñanza para las escuelas primarias y las preparatorias del profesor Manuel Antonio Rueda, (1931) Las cuatro operaciones de la aritmética, texto editado desde 1892 hasta 1944 en Colombia.

Con la enseñanza de la aritmética en la escuela primaria en Colombia, durante la primera mitad del siglo Xx, se hace visible la configuración de un saber escolar que dista mucho de los intereses de la disciplina de referencia, a saber, la matemática. Si bien el aprender las cuatro operaciones principales de la aritmética (sumar, restar, dividir y multiplicar) propiciaba en la escuela el acercamiento a los números, y mostraba un camino seguro para desarrollar las facultades intelectuales en los niños, en especial la atención y el razonamiento, la escuela utilizaba la aritmética como una estrategia para gobernar moralmente a los sujetos que formaba. La enseñanza de la aritmética termina por convertirse en una herramienta para configurar o reafirmar los diversos modos de constituirse como sujeto en la sociedad, naturalizada por medio del manual. Así lo intenta demostrar Bernardo Galindo (2014) en

carátulas del libro escolar, Biblioteca Virtual del Proyecto Relee, Redes de Estudios en Lectura y Escritura. Ministerio de Educación, Argentina. http://www.hum.unne.edu.ar/ investigacion/educa/web_relee/biblio.htm su informe sobre la enseñanza de las matemáticas en la escuela primaria en Colombia:

Es importante anotar que, dentro de los objetivos que le impusieron a la escuela primaria, estuvo el de ofrecer "a los educandos todo cuanto han menester para ser miembros útiles a la sociedad, a la familia y a sí mismos"; a lo anterior se agrega la idea de familia que se espera formar, donde se definen claramente los papeles que cada uno desempeña: "El rol del padre dentro de la familia, pues merced a su trabajo, la familia tiene pan, techo y vestido. Las labores de la madre: oficios caseros, cuidados morales y materiales respecto de los hijos y éstos dentro del círculo familiar reciben beneficios morales y materiales expresados en obligaciones que les corresponden como miembros de la familia". La Aritmética y la Matemática, como disciplinas escolares, no fueron ajenas a tal propósito y pusieron sus contenidos en función de los ideales modernos en los que el modelo de familia, de sociedad y sujeto constituyó las diversas prácticas sociales. No en vano los distintos discursos muestran el rango de practicidad que alcanzaron; pues a ella por el uso cotidiano que poseen, les quedaba más fácil serle útil a quien las aprendiera como condición para poder pertenecer a un marco social. En ese sentido, saber Aritmética o Matemática no estaba en discusión por su naturaleza de disciplina intelectual cuyo dominio provee de flexibilidad y destreza para moverse en la vida diaria; es desde allí que se le denomina como imprescindible necesidad para todos, "aún para aquellos individuos que por sus condiciones sociales han de ocupar los puestos de inferior categoría"; todos han de necesitar tanto de la aritmética como de la matemática; en virtud de lo anterior es que ese saber va a circular esgrimiendo como razón el uso cotidiano que ella presta y la utilidad que le otorga a quienes la aprenden, ya sea para las artes, la industria y otros muchos usos de la vida práctica; luego, a cada quien se le enseña la aritmética que necesita, dependiendo del oficio o la actividad que realice.

Puestas así las cosas, la Aritmética y la Matemática muestran la relación entre los sujetos, qué hace cada quien y cómo lo hace; en otras palabras, muestran unas maneras de ser de los sujetos en los distintos espacios de la vida cotidiana; para ello las imágenes, los problemas, sus intencionalidades y todos los discursos, nos han de servir para ver cómo dichas disciplinas coadyuvaron a configurar el tipo de subjetividad que fue pensado para ese momento. (2014, pp. 96, 97)

Finalmente, con la filosofía nos encontramos ante una práctica de enseñanza que desarrolla un proceso de transición y transformación de su estatuto, de ciencia universitaria a disciplina escolar. Sobre esta 
transformación de la filosofía enseñada en Colombia a lo largo del siglo XIX el profesor Saldarriaga sostiene:

Tal episodio podría describirse como el cambio de estatuto de la filosofía, en su paso de ciencia universitaria a disciplina escolar. En efecto, se puede establecer una periodización marcada por dos momentos: la situación de la filosofía a fines del siglo XVIII, cuando ella es una ciencia universitaria, con dos características: en lo institucional, se aloja en las Facultades Menores, como ciencia preparatoria - fundamento- para el estudio de las facultades mayores de teología, derecho, jurisprudencia y medicina; y en lo epistemológico, está constituida como "la ciencia de las ciencias", pues el canon de sus tres grandes tratados - Lógica, Metafísica y Ética- provee el método, los principios y los contenidos básicos del saber letrado universitario requerido por la República de las Letras. Y la situación de finales del siglo XIX, marcada por la organización del bachillerato "moderno", cuando la filosofía se relocaliza como una asignatura de nivel intermedio, coronamiento de la formación media en Humanidades. Y aunque sigue justificándose como un paso preparatorio para las ciencias universitarias, y aunque - en Colombia - conserva la estructura del canon humanista, ya no es la "ciencia de ciencias" sino un curso diseñado con criterios pedagógicos para los jóvenes bachilleres: es ya una disciplina escolar. En términos de D. Julia, "se pasa del viejo marco de la enseñanza humanista al modelo enciclopédico del siglo XIX en el que las disciplinas se imparten las unas al lado de las otras por profesores especialmente formados". (2015, p. 1)

A lo que agrega más adelante:

Lo que es significativo para esta historia de la filosofía como disciplina escolar, desde el punto de vista de los textos, es que no puede decirse que estemos ante una relación entre una "disciplina de referencia" y una "disciplina escolar", sino dentro de una "disciplina escolar" (ocupando lugar tanto en Universidades como en Colegios o Liceos) que produce ella misma sus propias jerarquías de textos. Por las declaraciones de Destutt, podríamos decir que la Ideología — con su canon completo- era ya una disciplina escolar porque fue escrita para los Colegios como un proyecto político de formación juvenil, si no fuera porque los mismos volúmenes de los Elementos también circularon como libros filosóficos en las Academias (en las de Ciencias Morales y Políticas hubo una sección denominada "Análisis de las ideas"), y Destutt nunca fue catalogado como profesor, sino como filósofo. Descubrimos una nueva faceta del régimen de verdad de la Filosofía como Pedagogía, en el que la circulación no se da en un solo sentido, Universidad-Colegio o Colegio-
Universidad (ya esto último es notable), sino en una doble dirección que busca pedagogizar la filosofía más allá fuera de la institución escolar, a la vez que escolariza la Filosofía como instrumento de enseñanza, y todo ello para una política del saber. Pero, eso no significa disolución de las diferencias entre los espacios institucionales, pues el método es el que mantiene, en la mejor tradición de Port Royal, la diferencia entre el método - y el momento- de indagación y el método y momento de exposición. Pero esta distinción no conlleva, aún, a un afuera y un adentro de la escuela como espacialización para el saber filosófico" (2015, p. 16)

\section{Cuestiones de método}

Este proyecto se inscribe en los análisis históricos sobre la educación y la pedagogía en Colombia, realizados por el Grupo de Investigación Historia de la Práctica Pedagógica en Colombia (GHPP), el cual adoptó el método arqueológico de Michel Foucault, no como herramienta teórica sino histórica para analizar los distintos saberes y prácticas sobre educación y pedagogía apropiadas en nuestro país desde el siglo XVIII hasta el siglo Xx. Con la arqueología, los sucesos de creación, los documentos y los discursos se asumen como acontecimientos de saber. Con la perspectiva de la arqueología del saber no se trata de buscar precursores, orígenes ni influencias entre autores, escuelas o ideas, ni de describir cuál de los sistemas ha sido el más verdadero, o el primero o el fundamental. En las expresiones del mismo Foucault:

No se tratará de conocimientos descritos en su progreso hacia una objetividad en la que, al fin, puede reconocerse nuestra ciencia actual; lo que se intentará sacar a luz es el campo epistemológico, la episteme en la que los conocimientos, considerados fuera de cualquier criterio que se refiera a su valor racional o a sus formas objetivas, hunden su positividad y manifiestan así una historia que no es la de su perfección creciente, sino la de sus condiciones de posibilidad. (1972, p. 7)

En este sentido, la noción metodológica más importante que suscita el análisis arqueológico es la de campo de saber. Un campo de saber, al que Foucault también denomina positividad, sería

... el dominio constituido por los diferentes objetos que adquirirán o no un estatuto científico [...] el espacio en el que el sujeto puede tomar posición para hablar de los objetos de que trata en su discurso[...] el campo de coordinación y de subordinación de los enunciados en que los conceptos aparecen, se definen, se aplican y se transforman [...] en fin, un saber se define por las posibilidades de utilización y 
apropiación ofrecidas por el discurso [...] Existen saberes que son independientes de las ciencias (que no son su esbozo histórico ni su reverso vivido), pero no existe saber sin una práctica discursiva definida, y toda práctica discursiva puede definirse por el saber que forma. (Foucault, 1996, pp. 306-307)

Desde esta perspectiva el proyecto analiza el proceso de configuración histórica de la enseñanza de las ciencias y los saberes en Colombia tomando como herramientas conceptuales y metodológicas la memoria activa del saber pedagógico, la práctica pedagógica, el saber pedagógico y la apropiación.

Con relación a la educación y la pedagogía, la noción de memoria activa del saber pedagógico se entiende como “... el lugar donde podemos encontrar cuáles han sido las formulaciones, las búsquedas, los fracasos, los obstáculos, las continuidades y los avances acerca de la enseñanza..." (Zuluaga, 2005), así como las discontinuidades, los discursos, las normas, los reglamentos, los manuales de enseñanza, los textos escolares, las disposiciones del cuerpo, los materiales de memoria (objetos, emblemas, pupitres, uniformes), que visibilizan y permiten reconstruir la historia de nuestras prácticas pedagógicas.

Para Zuluaga, la memoria activa se reconstruye gracias a un trabajo histórico donde también se descubren exclusiones, diferencias, estratificaciones, sometimientos, ocultamientos. Esas condiciones históricas están inscritas en los conceptos producidos. El término memoria activa indica la vigencia de conceptualizaciones y problemas para la actualidad, pero en ningún momento desliga la producción de conceptos de sus condiciones de posibilidad (Zuluaga, 2005). En este sentido se pueden leer las siguientes expresiones:

\begin{abstract}
Si bien las argumentaciones que Comenio externa en su Didáctica Magna en relación con las grandes etapas por las que ha de pasar una propuesta metodológica no son vigentes en este momento, una lectura de ellas nos permitiría visualizar el significativo valor que podrían tener en nuestros días, sobre todo por la sencillez de su elaboración. Los desarrollos de la psicología, la sociología, el pensamiento grupal, la ciencia de la comunicación, la tecnología y la didáctica nos colocan en otra situación de construcción metodológica muy distinta de la que existía en el siglo XVII o en el XIX, pero los grandes postulados comenianos, respecto al papel que cumple el método en las propuestas didácticas, conservan aún su vigencia para el trabajo educativo. (Díaz Barriga, 1997, pp.111-112).
\end{abstract}

Siguiendo con Zuluaga, la cuestión es de doble vía, es decir, el distanciamiento de condiciones de posibilidad significan una transformación histórica que puede ser una discontinuidad y la vigencia para el presente que cuestione, descubra, evidencie la pertinencia de problematizaciones y conceptos en la actualidad de un saber (Zuluaga, 2005).

Por su parte, el término práctica pedagógica designa:

1. Los modelos pedagógicos tanto teóricos como prácticos utilizados en los diferentes niveles de la enseñanza.

2. Una pluralidad de conceptos pertenecientes a campos heterogéneos de conocimiento retomados y aplicados por la pedagogía.

3. Las formas de funcionamiento de los discursos en las instituciones educativas donde se realizan prácticas pedagógicas.

4. Las características sociales adquiridas por la práctica pedagógica en las instituciones educativas de una sociedad dada que asigna unas funciones a los sujetos de esa práctica.

5. Las prácticas de enseñanza en diferentes espacios sociales, mediante elementos del saber pedagógico. (Zuluaga, 1999, p. 147)

Así explicada, esta noción permite comprender la pedagogía como teoría y práctica, como lo que ayuda a los maestros entrar en relación con el conocimiento, y como acontecimiento social susceptible de ser descrito en su especificidad histórica. Vale la pena recordar que nuestra problematización de la pedagogía, tan importante para la reflexión sobre las disciplinas y saberes escolares, se apoya en dos ejes:

Uno, la redefinición polémica de pedagogía como práctica pedagógica, esto es, no como sinónimo del quehacer empírico del maestro en el día tras día, sino como el conjunto de relaciones de saber y poder que inscriben los gestos del enseñar en cierta organización social de los saberes y de la cultura, relaciones que metodológicamente rastreamos en el juego de cuatro dimensiones: institución-sujetodiscurso-estrategia. En esto nos nutrimos de la caja de herramientas de la arqueología del saber y la genealogía del poder de Michel Foucault, que permite analizar los sistemas de producción de verdad y de gobierno de sujetos en formaciones sociales singulares (Zuluaga, 1999). Nos sostenemos en un segundo eje, el de la historia epistemológica de los saberes, en la que el recurso a las fuentes pasadas del saber pedagógico no obedece a un prurito de erudición académica, sino a la certeza de que la historia es un laboratorio que, frente a la permanente exigencia de novedad y actualización con que se acosa sin tregua a los maestros, nos permite construir una batería de conceptos y de instrumentos para esa reconstrucción 
actual deseada de la pedagogía como saber que, por un lado, fuese un pensamiento potente para hacer emerger las voces de los sujetos subalternizados en la escuela -estudiantes y maestros- y por otro, un pensamiento menor, es decir, un saber de las “pequeñas cosas", riguroso sí, pero sin pretensión hegemónica, más cercano a las artes del vivir que a las artes del gobernar. En luchas de largo aliento, la leyenda de David y Goliat nos ha enseñado que más vale construir pequeñas hondas que manejar pesadas armaduras. (Saldarriaga, 2008, p. 75)

¿Los saberes y disciplinas escolares son una dimensión del saber pedagógico? En este proyecto trabajamos con la idea que los saberes escolares son una dimensión del saber pedagógico, no lo desplaza, ya que es el conocimiento que más da cuenta de las prácticas de enseñanza. El saber escolar como una dimensión del saber pedagógico funciona como un flujo de relaciones con el afuera y el adentro de la escuela. De ahí lo estratégico para el proyecto utilizar la noción de saber pedagógico de Zuluaga entendido como

... el conjunto de conocimientos con estatuto teórico o practico que conforman un dominio de saber institucionalizado el cual configura la práctica de la enseñanza y la adecuación de la educación en una sociedad. Está conformado por los diferentes discursos a propósito de la enseñanza entendida en sentido práctico o conceptual. Reúne los temas referentes a la educación, la instrucción, la pedagogía, la didáctica y la enseñanza. (Zuluaga, 1999, p. 149)

El saber es el espacio más amplio y abierto de un conocimiento, donde se pueden localizar discursos de muy diferentes niveles, desde los que apenas empiezan a tener objetos de discursos y prácticas para diferenciarse de otros discursos y especificarse, hasta aquellos que logran una sistematicidad que todavía no obedece a criterios formales.

Al reflexionar sobre la enseñanza de los saberes y las disciplinas en la escuela colombiana desde la noción de saber acuñada por Foucault, se pretende abrir la pedagogía a las distintas relaciones con los demás saberes y ciencias, y estudiarla más allá de los territorios de la ciencia. Estudiar la pedagogía como ciencia le obligaría a establecer fronteras, estudiarla como saber le exige romper fronteras, establecer nuevas relaciones, y le obliga a dar el salto de la unicidad a la pluralidad. De acuerdo con esto, el saber pedagógico no es

... el nombre de alguna teoría que pretenda reemplazar los cuerpos teóricos conocidos bajo los nombres de pedagogía, educación o ciencias de la educación. Tampoco es equiparable a un amontonamiento caótico de toda clase de libros sobre estos temas. [...]
Saber pedagógico es, primero que todo, un concepto metodológico para reunir discursos a propósito de la enseñanza y la educación; es por tanto un conjunto pero de elementos dispares. [...] mientras una teoría tiene unidad, el saber constituye una pluralidad, [...] (Zuluaga, 2001, p. 83)

Como se trata de una investigación histórica sobre la configuración de los saberes y las disciplinas escolares para hacer visible las diversas formas de apropiación de las ciencias y los saberes en la enseñanza en Colombia, este proyecto reflexiona bajo el concepto de apropiación elaborado por la profesora Olga Lucia Zuluaga ${ }^{4}$. Con esta herramienta metodológica logramos obtener la potencia para problematizar el régimen de orden y verdad que ha distinguido lo propio de la modernidad, a saber: pensar que existe un campo de meras representaciones y otro real, un mundo de originales y modelos y otro de descripciones y copias. De ahí que en este proyecto no trabajemos desde el dualismo "saberes científicos/ saberes escolares", o más específicamente "matemáticas científicas/ matemáticas escolares", sino bajo la de "elementos comunes/ensamblajes diferentes" (Saldarriaga, 2005).

Así, tratando de resolver el desacompasamiento creciente, descrito por Feldman (2010) en las relaciones entre conocimiento escolar, dispositivos escolares, maestros y pedagogía, la noción de apropiación propone describir las interacciones complejas entre las diversas lógicas y temporalidades de los saberes científicos y escolares, acudiendo en términos de Saldarriaga, “a las metáforas del 'dispositivo' o de la 'bisagra' para hacer ver el funcionamiento de los ensamblajes conceptuales e institucionales ad hoc que resultan de tales interacciones" (2005, p. XX).

\section{Reflexiones finales y perspectiva de investigación}

Bien sabemos que el campo de la historia de las disciplinas y de los saberes escolares es un espacio de investigación de recientes problematizaciones en el área de la historia de la educación y la pedagogía en Latinoamérica, y un campo de estudios por abrirse en nuestro país. Los Congresos latinoamericanos sobre historia de la educación, han sido una oportunidad para que la comunidad académica nacional,

4 La apropiación desde Olga Lucía Zuluaga (1997) pretende, en primer lugar, describir el universo de lo apropiado, en otras palabras, lo que viene de afuera, identificando las reglas de su funcionamiento. En segundo lugar, analizar cómo y mediante que filtros se apropió lo foráneo, esto para ver los recortes, las semejanzas y las diferencias. 
intercambie los primeros esbozos de este campo de estudios sobre los contenidos de enseñanza de las ciencias, las disciplinas y los saberes en Colombia. En estos eventos académicos se ha venido abordando el objeto de una historia de las disciplinas y los saberes escolares, a saber: la historia de los contenidos de la enseñanza. De acuerdo con esto y en el contexto del presente proyecto es oportuno plantearnos algunas preguntas que nos permitan construir algunas reflexiones de cierre del presente artículo.

1. La primera es la planteada por Chervel hace más de dos décadas:

¿Tiene algún sentido la noción de historia de las disciplinas escolares? ¿Presenta analogías o nexos comunes la historia de las diferentes disciplinas? Y, para ahondar un poco más, ¿permite la observación histórica extraer normas de funcionamiento o incluso uno o varios modelos disciplinarios ideales, cuyo conocimiento y aplicación podrían ser de alguna utilidad en los debates pedagógicos presentes y futuros? (1991, p. 59)

En efecto, con este trabajo de investigación se aportan algunas reflexiones que permitan hacer visible los siguientes aspectos: a) construir un campo de conocimientos sobre la escuela, la enseñanza de las ciencias, las disciplinas y los saberes cuyo conocimiento y aplicación podrían ser de alguna utilidad en los debates actuales sobre la relación entre la pedagogía y las ciencias en el marco de la formación de los maestros en Colombia; b) aporta elementos teóricos y metodológicos acerca de la relevancia de la construcción y estudio de las "disciplinas y saberes escolares" para señalar cómo el saber escolar contribuye a la cultura en general, a pesar del bajo perfil que por lo general se le concede a dicho saber. En consecuencia, c) muestra el crucial lugar que ocupa el maestro en esta historia, no solo por ser protagonista de primera línea, en tanto mediador cultural, sino porque sigue desempeñando un papel importante en la definición de las prácticas de enseñanza.

2. ¿Los saberes escolares son una construcción en relación con el saber pedagógico, es decir, se han construido desde las prácticas mismas, ellos han producido teoría y prácticas?

Como lo habíamos dicho, este proyecto trabaja con la idea que los saberes escolares son una dimensión del saber pedagógico, no lo desplaza, ya que es el conocimiento que más da cuenta de las prácticas de enseñanza. El saber escolar como dimensión del saber pedagógico funciona como un flujo de relaciones con el afuera y el adentro de la escuela. Tal funcionamiento interno y externo permite que los saberes escolares actúen como prácticas y no como teorías totalizadoras. Lo que se propone en este proyecto es examinar los saberes escolares desde las nuevas maneras de entender las relaciones entre teoría y práctica, es decir, no desde el prisma de un proceso de totalización en un sentido o en otro, sino desde un horizonte mucho más parcial y fragmentario. Siguiendo a Deleuze, "una teoría es siempre local, relativa a un campo pequeño, y puede tener su aplicación en otro dominio más o menos lejano" (Foucault, 1999). Teniendo en cuenta que esta relación de aplicación nunca es de semejanza. En este sentido, los saberes escolares no son nuevas teorías que expresan y traducen las prácticas de enseñanza, son una práctica local y regional, no totalizadora del saber pedagógico.

3. ¿Las nuevas formas de apropiación de lo científico en la escuela, que cita el proyecto de John Dewey, para hacer visible la configuración de los saberes y las disciplinas escolares en Colombia, darían cuenta de una trasposición didáctica, o no son tema de interés?

No es que no sea un tema de interés sino que la pregunta sobre la configuración de los saberes y disciplinas escolares en el saber pedagógico colombiano van más allá de la transposición didáctica, ya que no se trata de ver el paso del saber sabio al enseñado, ni de ver en las prácticas de enseñanza un saber subordinado a las disciplinas o ciencias de referencia. En términos arqueológicos, las disciplinas escolares o saberes escolares no se hallan en un estado de subordinación teleológica en relación con la ortogénesis de las ciencias (Foucault, 1996).

De lo que se trata, y he aquí la apuesta del proyecto, es de analizar el estatuto de los saberes y disciplinas escolares. Un estatuto que no es mediado por la ya clásica relación entre los saberes sabios y los saberes enseñados, sino por las relaciones que establece Dewey en El niño y el programa escolar (1999), entre el maestro y el científico respecto de la materia de estudio. Relación que lo lleva a distinguir, no oponer, las prácticas científicas y las prácticas de enseñanza. Las formas de apropiación de los saberes y las disciplinas en la escuela están inspiradas más en esta relación que en la establecida por el francés Chevallard.

Las disciplinas y saberes escolares ya no se rigen por la legitimidad epistemológica dada por la disciplina científica en que se origina, su legitimidad social y cultural es dada por la práctica misma de enseñanza, la cual tiene una naturaleza y caracterización muy distinta a la elaborada por las didácticas disciplinares. 
En el marco de la problematización de este objeto de estudio el proyecto aporta algunas reflexiones para la configuración de este prometedor campo de estudios sobre los saberes y las disciplinas escolares en Colombia, ya que se ocupa de analizar las diversas relaciones que establecen entre enseñanza, ciencias y saberes en el saber pedagógico colombiano, haciendo visible la configuración de un saber sobre la enseñanza de las ciencias y los saberes en las escuelas primaria y secundaria. Queda por analizar en futuras investigaciones cómo se establece la relación o qué nexos se configuran entre la historia de los saberes y las disciplinas escolares con la historia de las diferentes disciplinas y ciencias.

\section{Referencias bibliográficas}

Álvarez Gallego, A. (2013). Las ciencias sociales en Colombia. Genealogías pedagógicas. Bogotá: IDEP, Alcaldía Mayor de Bogotá.

Castro, E. (2004) El vocabulario de Michel Foucault. Buenos Aires: Universidad Nacional de Quilmes.

Chervel, A. (1991, mayo-agosto). Historia de las disciplinas escolares. Reflexiones sobre un campo de investigación. Revista de Educación 295, 59-111.

Cucuzza, R. H. (2008). Retórica de las escenas de lectura en las carátulas del libro escolar, Biblioteca Virtual del Proyecto Relee, Redes de Estudios en Lectura y Escritura. Argentina: Ministerio de Educación.

Dewey, J. (1984). The sources of a science of education 1929. En J. Sáenz (trad.). The later works, volume 5: 1929-1930. pp. 1-40. Carbondale: Southern Illinois Press.

Dewey, J. (1999). El niño y el programa escolar. Buenos Aires: Losada.

Díaz Barriga, A. (1997). Lo metodológico: tema central del debate didáctico y la responsabilidad docente. En A. Díaz Barriga. Didáctica y currículum. México: Editorial Paidós.

Durkheim, E. (1992). Historia de la educación y de las doctrinas pedagógicas. La evolución de la pedagogía en Francia. Madrid: La Piqueta.

Feldman, D. (2010). Estados alterados en la escuela. Lo que cambia y lo que permanece. En G. Frigerioy G. Diker. Educar: saberes alterados. Buenos Aires: Del estante, Clacso.

Foucault, M. (1972). Las palabrasy las cosas. Una arqueología de las ciencias humanas. México: Siglo XXI editores.

Foucault, M. (1995). La verdad y las formas jurídicas. Barcelona: Gedisa.

Foucault, M. (1996). Arqueología del saber. México: Siglo xxI editores.
Foucault, M. (1999). Los intelectuales y el poder, Entrevista a Michel Foucault por Gilles Deleuze. En Estrategias de poder. Barcelona: Ediciones Paidós.

Galindo, B. (2014). La enseñanza de la aritmética y la matemática en Colombia. Primera mitad del siglo XX. Métodos, materiales y sujetos. Tesis de maestría en Educación, Universidad Pedagógica Nacional, Bogotá.

Herrera Restrepo, S. M. (2015). Un saber de frontera. La literatura como estrategia de formación de las emociones patriotas en Colombia. Informe final presentado al proyecto "Saberes y disciplinas escolares en Colombia". Colciencias, Universidad del Valle, Universidad de Antioquia, Universidad Pedagógica de Colombia, Pontificia Universidad Javeriana.

Julia, D. (2000). La construcción de las disciplinas escolares en Europa. En J. Ruiz Berrio (ed.). La cultura escolar de Europa. Tendencias históricas emergentes (pp. 45-78). Madrid: Biblioteca Nueva.

Martínez Boom, A. (1986). Escuela, maestro y métodos en Colombia 1750-1820. Bogotá: Universidad Pedagógica Nacional.

Quintana Marín, L. M. (2015). Sujetos, textos y prácticas: La enseñanza de la lectura y la escritura como saber escolar en Colombia 1889-1963. Informe final presentado al proyecto "Saberes y disciplinas escolares en Colombia". Colciencias, Universidad del Valle, Universidad de Antioquia, Universidad Pedagógica de Colombia, Pontificia Universidad Javeriana.

Sáenz, J.; Saldarriaga, O. y Ospina, A. (1997). Mirar la infancia: pedagogía, moral y modernidad en Colombia, 1903-1946. Volumen. 2. Medellín: Colciencias, Foro Nacional por Colombia, Uniandes, Universidad de Antioquia.

Saldarriaga, O. (2005). Nova et Vetera o de cómo fue apropiada la filosofía neotomista en Colombia, 1868-1930. Catolicismo, modernidady educación desde un país poscolonial latinoamericano. (Tesis doctoral). Facultad de Filosofía y Letras, Universidad Católica de Lovaina, Bélgica.

Saldarriaga, O. (2008). De la pedagogía al saber pedagógico: Notas para (un) saber del currículo. En Cuadernos de Psicopedagogía, 5.

Saldarriaga, O. (2015). La filosofía enseñada: cánones, instituciones, epistemes, Colombia, siglo Xx. Informe final presentado al proyecto Saberes y disciplinas escolares en Colombia. Colciencias, Universidad del Valle, Universidad de Antioquia, Universidad Pedagógica de Colombia, Pontificia Universidad Javeriana.

Rueda, M.A. (1931) Las cuatro operaciones de la Aritmética. Texto de enseñanza para las escuelas primarias y las preparatorias. Undécima edición, Bogotá: Librería Camacho Roldán y Compañía. 
Zuluaga, O. L. (1997). Prólogo. En J. Sáenz, O. Saldarriaga, y A. Ospina. Mirar la infancia: pedagogía, moral y modernidad en Colombia, 1903-1946. Volumen 1. Medellín: Colciencias, Foro Nacional por Colombia, Uniandes, Universidad de Antioquia.

Zuluaga, O. L. (1999). Pedagogía e historia. La historicidad de la pedagogía. La enseñanza, un objeto de saber. Bogotá: Siglo del Hombre Editores, Anthropos, Universidad de Antioquia.

Zuluaga, O. L. (2001). El saber pedagógico: experiencias y conceptualizaciones. En Encuentros pedagógicos transculturales. Desarrollo comparado de las conceptualizaciones y experiencias pedagógicas en Colombia y Alemania. Medellín: Universidad de Antioquia.

Zuluaga, O. L. (2005). Saber pedagógico y campos conceptuales. Proyecto de Investigación presentado al IDEP, Santa Fe de Bogotá.

Zuluaga, O. L. y Martínez Boom, A. (1997). Focalizando los manuales escolares desde las prácticas y los saberes. En: Segundo Seminario Internacional Textos Escolares en Iberoamérica "Avatares del pasado y tendencias actuales". Buenos Aires: Universidad Nacional de Quilmes. 